\title{
Strategic Human Rights Litigation - Eine Standortbestimmung
}

\author{
Vanessa Rüegger *
}

Menschenrechte bilden das Fundament der geltenden Rechtsordnung, sind Voraussetzung einer funktionierenden Demokratie und gewährleisten einen Minimalstandard im staatlichen Umgang mit Menschen. Die Schweiz weist generell einen hohen Schutzstandard auf. Politische und gesellschaftliche Herausforderungen setzen die Menschenrechte jedoch immer wieder unter Druck. Rechtliche und faktische Lücken im Rechtsschutz beschränken die Durchsetzung der Menschenrechte empfindlich und treffen gerade diejenigen Mitglieder der Gesellschaft am stärksten, welche am ehesten dieses Schutzes bedürften. Für die Stärkung des Menschenrechtsschutzes stehen der Zivilgesellschaft verschiedene Instrumente zur Verfügung. Dazu gehört neben Rechtsberatung und politischem Engagement auch das Instrument der strategischen Prozessführung. Strategic Human Rights Litigation (SHRL) hat sich in den vergangenen Jahren weltweit zu einem spezialisierten Tätigkeitsgebiet entwickelt. Der vorliegende Beitrag bietet einen ersten Überblick zum Thema im Kontext der Schweizer Rechtsordnung.

I. Einleitung. 95

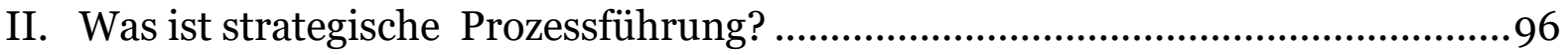

III. Warum braucht es strategische Prozesse? .......................................................99

IV. Wie werden strategische Prozesse organisiert? ............................................... 102

V. Welche Beispiele für strategische Prozesse gibt es? .......................................109

VI. Plädoyer für eine Stärkung der Menschenrechtspraxis ....................................112

Zitiervorschlag: Vanessa Rüegger, Strategic Human Rights Litigation - Eine Standortbestimmung, in: sui-generis 2020, S. 94

URL: $\quad$ sui-generis.ch/124

DOI: $\quad$ https://doi.org/10.21257/sg.124

* Dr. iur. Vanessa Rüegger (va.ruegger@gmail.com) ist Postdoc und Lehrbeauftragte an der Universität Basel und arbeitet momentan in einer Anwaltskanzlei in Zürich. Der vorliegende Beitrag ist im Rahmen ihres Forschungsaufenthalts 2018/19 als Fulbright Scholar an der Cardozo Law School sowie als Bernstein Human Rights Fellow an der NYU School of Law und ihrer Mitarbeit in der Arbeitsgruppe «Zugang zum Recht» von humanrights.ch und Schutzfaktor M entstanden

Dieses Werk ist lizenziert unter einer Creative Commons Namensnennung - Weitergabe unter gleichen Bedingungen 4.0 International Lizenz. 


\section{Einleitung}

1 Menschenrechte bilden das Fundament der Rechtsordnung, sind Voraussetzung einer funktionierenden Demokratie und gewährleisten einen Minimalstandard im staatlichen Umgang mit Menschen. ${ }^{1}$ Rechtliche und faktische Lücken im Menschenrechtsschutz treffen gerade diejenigen Mitglieder der Gesellschaft am stärksten, welche am ehesten dieses Schutzes bedürften. Grundsätzlich ist es die Pflicht des Staates, diesen Lücken mit positiven Massnahmen zu begegnen, wie beispielsweise die Anpassung der Rechtsordnung, subventionierter Rechtsberatung oder unentgeltlicher Rechtspflege. Staaten sind als Adressaten der Menschenrechte dazu verpflichtet, die Rechtsordnung und die Institutionen der Rechtsanwendung so auszugestalten, dass alle Menschen den gleichen Zugang zum Recht haben und ihre Menschenrechte effektiv einfordern können. Diese Verpflichtung beschränkt sich nicht auf die formelle Gewährleistung von rechtlichen Verfahren und Streitbeilegungsmechanismen. Staaten müssen sicherstellen, dass der Zugang zum Recht de facto

\footnotetext{
Für eine allgemeine Einführung in die Grundlagen des Menschenrechtsschutzes siehe Regina Kiener, Was sind Menschenrechte?, in: Frau Huber geht nach Strassburg, 2018, S. 13-23. Zur Bedeutung der Grundrechte in der Bundesverfassung einführend René Rhinow/Markus Schefer/ Peter Uebersax, Verfassungsrecht, 3. Aufl., 2016, N 951-980; Jörg Paul Müller, Elemente einer Grundrechtslehre, 1982, $1 \mathrm{ff}$.; Regina Kiener/ Walter Kälin, Grundrechte, 3. Aufl., S. 10; Andreas Kley, St. Galler Kommentar BV, N 8 ff. der geschichtlichen Einleitung; Dieter Grimm, Zukunft der Verfassung II, 2012, S. 16 ff. Zum internationalen Menschenrechtsschutz einführend Mashood A. Baderin/Manisuli Ssenyonjo, Development of International Human Rights Law before and after the UDHR, in: International Human Rights Law, 2010, S. 3-30; Hans Joas, Die Sakralität der Person, 2015, S. 23 ff.; Lynn Hunt, Inventing Human Rights, 2008.
}

tatsächlich gewährleistet ist. ${ }^{2}$ Aber auch der Zivilgesellschaft kommt eine wichtige Funktion für die Durchsetzung des Menschenrechtsschutzes zu. Zur Stärkung des Menschenrechtsschutzes stehen ihr verschiedene Instrumente zur Verfügung. Dazu gehört neben politischem Engagement, der Arbeit von NGOs und spezialisierten Rechtsberatungsstellen auch das Instrument der strategischen Prozessführung.

2 Strategic Human Rights Litigation (SHRL) hat sich in den vergangenen Jahren zu einem spezialisierten Tätigkeitsgebiet entwickelt, an dem eine Vielzahl von Akteuren beteiligt sind. Das Instrument der strategischen Prozessführung wird auch in der Schweiz zunehmend bewusst eingesetzt. Der vorliegende Beitrag greift diese Entwicklung auf und bietet eine Standortbestimmung über die strategische Prozessführung im Bereich der Menschenrechte im Kontext der Schweizer Rechtsordnung. Der Aufsatz thematisiert nicht eine dogmatische Fragestellung, sondern beobachtet die Ausdifferenzierung einer spezialisierten Praxis im Bereich des Menschenrechtsschutzes. Die Untersuchung basiert auf einem

$2 \overline{\text { Die Verpflichtungen des Staates werden in der }}$ Literatur unter den Stichworten Zugang zum Recht, Zugang zur Justiz oder access to justice diskutiert, siehe einführend Deborah L. Rhode, Access to Justice, 2004; für die Schweiz grundlegend Regina Kiener, Zugang zur Justiz, ZSR 138/2019 II S. 7-99; Claudia Kaufmann/Christina Hausamann, Zugang zum Recht, 2017; zur Diskussion im amerikanischen Kontext vgl. etwa die Beiträge in der Zeitschrift Dædalus, Access to justice, 148 (2018) 1; siehe auch Report of the Special Rapporteur on extreme poverty and human rights (UN Doc. A/67/278) (9. August 2012); UNDP, Access to justice, Practice Note (3. September 2004). Für eine quantitative Analyse der Situation in 45 Ländern weltweit siehe World Justice Project, Global Insights on Access to Justice, 2018. 
qualitativen Forschungsansatz. Da die strategische Prozessführung im Bereich der Menschenrechte erst ansatzweise erforscht ist, stützt sich die Untersuchung - neben Beiträgen aus der Lehre und ausgewählten Beispielen aus der Rechtsprechung - auf Gespräche mit Akteuren aus dem Fachgebiet in den USA, in Deutschland und in der Schweiz, welche ich als Fulbright Scholar im Rahmen des Forschungsprojekts Learning about Human Rights Clinics and Litigation in den Jahren 2018 und 2019 geführt habe. 3 Der Beitrag soll dieses im Austausch mit der Praxis gesammelte Wissen leicht zugänglich und übersichtlich darstellen. Für eine vertiefte Auseinandersetzung wird auf die bisweilen vereinzelt vorhan-

3 Ich danke allen, die mir in zahlreichen Interviews über ihre Erfahrungen im Bereich Human Rights Litigation berichtet haben. Mein Dank gilt Jocelyn Getgen Kestenbaum, Professorin und Direktorin der Benjamin B. Ferencz Human Rights and Atrocity Prevention Clinic an der Cardozo Law School und Meg Satterthwaite, Professorin und Direktorin der Global Justice Clinic an der NYU School of Law ebenso wie Stephan Bernard, Andrea Huber, Marianne Aeberhard und David Mühlemann von der Arbeitsgruppe Zugang zum Recht bei humanrights.ch sowie allen Teilnehmerinnen und Teilnehmer der von der Arbeitsgruppe organisierten Roundtables zur Praxis des Menschenrechtsschutzes (April 2019). Ganz besonders danke ich Burt Neuborne, Professor und Legal Director des Brennan Center for Justice der NYU und ehemaliger National Legal Director der ACLU, Steven R. Shapiro, Professor an der NYU und ehemaliger Legal Director der ACLU, James A. Goldstone, Executive Director der OSJF, dass ich 2018/19 an ihren Vorlesungen an der NYU teilnehmen durfte und sie mir bereitwillig Auskunft gaben über ihre Erfahrungen im Bereich der strategischen Prozessführung. Besonderer Dank gilt auch David Cole, National Legal Director der ACLU, Bryan Stevenson, Professor an der NYU und Direktor der Equal Justice Initiative, Susanne Baer, Richterin am Bundesverfassungsgericht und Professorin an der Humboldt Universität in Berlin, Franz Werro, Professor an der Universität Fribourg und Georgetown Law, und Markus Schefer, Professor an der Universität Basel und Mitglied des UNO-Ausschusses über die Rechte von Menschen mit Behinderungen. dene Fachliteratur verwiesen. Der vorliegende Aufsatz konturiert den Begriff der strategischen Prozessführung, befasst sich mit den Gründen, warum strategische Prozesse geführt werden, zeigt die Organisationsstruktur strategischer Prozesse auf und stellt konkrete Fälle strategischer Prozessführung vor. Im letzten Abschnitt verbindet die Arbeit die Entwicklungen im Bereich der strategischen Prozessführung mit weiteren Veränderungen im Rechtsgebiet und schliesst mit einem Plädoyer für die Stärkung der Menschenrechtspraxis.

\section{Was ist strategische Prozessführung?}

3 Zunächst einmal stellt sich die Frage, was unter dem Begriff der strategischen Prozessführung im Bereich der Menschenrechte gemeint ist und inwiefern sich strategische Prozesse von gewöhnlichen Prozessen unterscheiden. Die Auseinandersetzung mit dieser Abgrenzung zwingt zugleich zur Thematisierung von Funktion und Grenzen strategischer Prozessführung.

\section{Begriff}

4 Im Englischen bezeichnet Human Rights Litigation die gewöhnliche Prozessführung zum Schutz der Menschenrechte einer Person oder Gruppe ohne weitere, begleitende Strategien. Strategic Human Rights Litigation (teilweise auch als public interest litigation oder impact litigation bezeichnet) ist demgegenüber ein spezifisches Instrument zur Stärkung des Menschenrechtsschutzes, mit dem bei Rechtsstreitigkeiten ein Ziel verfolgt wird, das weiter gefasst ist als die Interessen der unmittelbar involvierten Parteien. Strategische Prozessführung nutzt 
die Gerichte, um die Menschenrechte gezielt in einer Weise zu fördern, die über die Partikularinteressen der Opfer im jeweiligen Fall hinausgeht.4 Durch strategische Prozessführung können Menschenrechtsanliegen einer Person in strategischer Weise dazu dienen, strukturelle Menschenrechtsverletzungen zu thematisieren.

Der strategischen Prozessführung ist in den vergangenen zwanzig Jahren zunehmende Aufmerksamkeit zugekommen. Das Instrument findet mittlerweile in verschiedenen Rechtsordnungen breite Verwendung. Strategische Prozessführung wird neben Verfahren vor nationalen Gerichten auch dazu verwendet, Fälle vor internationalen Gerichten wie dem Europäischen Gerichtshof für Menschenrechte und dem Interamerikanischen Gerichtshof für Menschenrechte und vor internationale Gremien wie etwa den UN-Menschenrechtsausschuss zu bringen. Dieser Entwicklung kommt seit einigen Jahren auch in der Literatur vermehrte Aufmerksamkeit zu. 5

Helen Duffy, Strategic Human Rights Litigation, 2018, S. 3.

5 Aus der Literatur zum Thema siehe insbes. Helen Duffy (Fn. 4); Open Society Justice Initiative, Strategic Litigation Impacts, 2018; Alexander Graser /Christian Helmrich (Hrsg.), Strategic Litigation, 2019; Jules Lobel, Success Without Victory, 2004; Aryeh Neyer, Only Judgment: The Limits of Litigation in Social Change, 2012; David Cole, Engines of Liberty, 2017; Jackie Dugard/ Malcolm Langford, Art or Science? Synthesising lessons from public interest litigation, South African Journal on Human Rights 27/2011, S. 39-64; César Rodriguez-Garavito, Beyond the courtroom, Texas Law Review 89/2011, S. 1669-1698; Steven Budlender/Gilbert Marcus/Nick Ferreira, Public Interest Litigation and Social Change in South Africa, 2014; Richard J. Wilson/Jennifer Rasmussen, Promoting Justice: A Practical Guide to Human Rights Lawyering, 2001; Mark Tushnet, The NAACP's Legal Strategy against Segregated Education, 2005; Michael J. Klarman,

\section{Funktion der strategischen Prozessführung}

6 Nach Helen Duffy, Autorin des momen$\tan$ wegweisenden Werkes zum Thema, ist es in der Praxis schwierig, gewöhnliche Prozesse von strategisch geführten Prozessen zu unterscheiden, denn jeder Gerichtsfall im Bereich der Menschenrechte trägt unweigerlich immer beide Facetten in sich. Auch ein Menschenrechtsfall, mit dem die Parteien und ihre Vertretung einzig die Feststellung einer Menschenrechtsverletzung im Interesse der Beschwerdeführerin oder des Beschwerdeführers verlangen, hat eine kollektive Dimension, weil Menschenrechtsfragen immer den Kern der Rechtsordnung und also die normative Ausrichtung einer Gesellschaft betreffen. ${ }^{6}$ Duffy ist deshalb der Ansicht, dass die Unterscheidung zwischen gewöhnlicher und strategischer Prozessführung vermieden werden sollte.

7 Meiner Ansicht nach ist die Unterscheidung zwischen gewöhnlich und strate-

From the Closet to the Altar, 2014; Michael Clark, Public Interest Legal Services in South Africa Project Report, 2015; Anuj Bhuwania, Courting the People: Public Litigation in Post-Emergency India, 2017; Ivana Bacik/Mary Rogan, Legal Cases that Changed Ireland, 2016; Stuart Scheingold/Austin Sarat, Something to Believe In: Politics, Professionalism, and Cause Lawyering, 2004; ERRC, Strategic Litigation of Race Discrimination in Europe (2004); COHRE, Litigating Economic, Social and Cultural Rights, 2003; Byron Sheldrick, Blocking Public Participation: The Use of Strategic Litigation to Silence Political Expression, 2014; Gesine Fuchs/Dia Anagnostou/Susan Millns, Strategic Litigation for Gender Equality in the Workplace and Legal Opportunity Structures in Four European Countries, Canadian Journal of Law and Society, 28 (2013) 2, 189-208; ausserdem besonders empfehlenswert ist der persönliche Erfahrungsbericht von Bryan Stevenson, Just Mercy, 2015 (unter demselben Titel auch verfilmt).

6 Duffy (Fn. 4). 
gisch geführten Menschenrechtsprozessen aber gerade deshalb hilfreich, weil sie die Aufmerksamkeit auf die unterschiedlichen Dimensionen eines Menschenrechtsfalles lenkt. Menschenrechtsfragen sind persönlicher, aber auch gesellschaftlicher Art. Jeder Menschenrechtsfall hat aufgrund der besonderen Stellung der Menschenrechte als Fundament der Rechtsordnung zusätzlich zu seiner privaten immer auch eine politische Dimension. Einen Rechtsfall als strategische Chance für die Thematisierung eines breiteren Anliegens zu erkennen und mit einer über den Einzelfall hinausweisenden Strategie zur Stärkung des Menschenrechtsschutzes für die gesamte Gesellschaft zu verbinden, verändert jedoch unweigerlich die rechtliche und politische Tragweite eines Falles.

Die Unterscheidung zwischen gewöhnlicher und strategischer Prozessführung zwingt auch dazu, die Funktion der Menschenrechte in einer demokratischen Verfassungsordnung und die Funktionsaufteilung zwischen rechtsetzenden und rechtsanwendenden Organen bei jeder Menschenrechtsfrage mitzudenken. Die zentrale Funktion der Menschenrechte ist es, die vom verfassungsgebenden Organ als besonders wertvoll anerkannten Rechtsgüter des Einzelnen vor staatlichen Eingriffen und gegenüber demokratischen Mehrheitsentscheiden zu schützen. Den Gerichten kommt als rechtsanwendendes Organ die Funktion zu, die Menschenrechte als rechtsstaatliche Garantien auch gegenüber dem Gesetzgeber zu gewährleisten. Gerichtliche Urteile zum Schutz der Menschenrechte sind im Rahmen dieser Kompetenz deshalb nicht anti-demokratisch, sondern gerade Ausdruck einer funktionierenden
Verfassungsordnung, die nach dem Prinzip der Gewaltenteilung aufgebaut ist. Giacometti bezeichnete die Demokratie als Hüterin der Menschenrechte. Aber die Menschenrechte sind zugleich Hüterin der Demokratie und ihre gerichtliche Durchsetzung ein Beitrag zu deren Stärkung.

9 Dabei ist es jedoch wichtig zu berücksichtigen, dass Menschenrechte meist offen formulierte Bestimmungen sind, die den Gerichten einen weiten Spielraum in der Konkretisierung der Grundrechte belassen. Diese Offenheit ist nicht ein Defizit der Menschenrechte, sondern Ausdruck ihrer Funktion im Rahmen der geltenden Verfassungsordnung. Während verfassungsrechtliche Organisationsbestimmung oder technische Rechtssätze den Gerichten wenig Beurteilungsspielraum einräumen, ja diesen von vornherein beschränken sollen, sind Gerichte bei der Anwendung der Menschenrechte gerade dazu aufgefordert, sich vertieft und eigenständig mit den grundlegenden Wertungs- und Abwägungsfragen auseinanderzusetzen, welche menschenrechtliche Problemstellungen aufwerfen.7 Menschenrechtsfälle strategisch zur Thematisierung von grundlegenden Rechtsfragen zu nutzen bedeutet in dieser Hinsicht, auf die effektive Tragweite von Menschenrechtsfällen aufmerksam zu machen und ihr

\footnotetext{
Grundsätzlich Hans Huber, Über die Konkretisierung der Grundrechte, in: Der Staat als Aufgabe, 1972, S. 199, 204 f.; Jörg Paul Müller, in: HGR VII/2, § 202 N 21; Dieter Grimm, Recht und Staat der bürgerlichen Gesellschaft, 1987, S. 309; des Weiteren auch Pierre Tschannen, Die Auslegung der neuen Bundesverfassung, in: Neue Bundesverfassung, 2000, S. 236 f.; Walter Haller, Verfassungsfortbildung durch Richterrecht, ZSR 1/2005, S. 10-12; Eva Maria Belser, in: Basler Kommentar BV, N 67 zu Einleitung; Kiener/Kälin (Fn. 1), S. 69; Rhinow/Schefer/ Uebersax (Fn. 1), N 1091.
} 
Potenzial zum Schutz der Verfassungsordnung auszuschöpfen.

\section{Grenzen der strategischen Prozessführung}

10 Die besondere Herausforderung von strategisch geführten Menschenrechtsfällen besteht darin, die Funktion der Menschenrechte und der Gerichte als rechtsanwendendes Organ weder zu überschätzen noch zu unterschätzen. Der Begriff der strategischen Prozessführung ist insofern hilfreich, als er einerseits die gesamtgesellschaftliche Bedeutung von Menschenrechtsfällen erkennen lässt, zugleich aber dazu zwingt, ein Bewusstsein für die beschränkte normative Wirkung der Menschenrechte und die begrenzte Kompetenz der Gerichte als rechtsanwendendes Organ zu entwickeln. Menschenrechte sind das Rückgrat der Verfassungsordnung, aber sie sind nicht die gesamte Rechtsordnung. Sie machen der Legislative wichtige Vorgaben, überlassen den rechtsetzenden Organen aber in vielen Bereichen den Entscheid darüber, wie sie die Rechtsordnung im Detail ausgestalten. Die Gerichte haben ihrerseits die wichtige Funktion, Individualansprüche des Einzelnen durchzusetzen und dabei die Entscheide der Legislative auf ihre Übereinstimmung mit den Menschenrechten zu prüfen. Die gerichtliche Prüfungsfunktion ist damit aber auch eng begrenzt. Es liegt in der Regel nicht in der Kompetenz der Gerichte, die regulativen Kompetenzen der Legislative zu beschränken oder rechtsetzende Entscheide anstelle des Gesetzgebers vorzunehmen.

Für die Prozessführung in Menschenrechtsfällen bedeutet das, dass das strategische Potenzial von Menschenrechts- fällen in ihrer in jedem Einzelfall vorhandenen Tragweite als Kern der Rechtsordnung ausgeschöpft werden darf und soll. Über den gerichtlichen Weg nicht erzwungen werden können demgegenüber Anliegen, die in die Kompetenz der rechtsetzenden Behörden fallen, wobei die Abgrenzung nicht immer eindeutig ausfällt. Jede strategische Prozessführung, die einen gewöhnlichen Menschenrechtsfall mit einem über den Einzelfall hinausweisenden Ziel verbindet, muss sich deshalb eingehend damit befassen, ob die inhaltlichen Vorbringen tatsächlich in den normativen Geltungsbereich eines Menschenrechts und ihre Gewährleistung in den gerichtlichen Kompetenzbereich fallen oder nicht vielmehr dem Gesetzgeber zur Konkretisierung überlassen sind und auf dem politischen Weg eingefordert werden müssen. Strategische Prozessführung ist ein Instrument neben anderen, um den Menschenrechtsschutz zu verbessern. Strategic Human Rights Litigation ist nicht für alle Menschenrechtsfragen die passende Lösung und sollte niemals als solche mystifiziert werden. ${ }^{8}$

\section{Warum braucht es strategische Prozesse?}

12 Verschiedene Gründe sprechen dafür, Menschenrechtsfälle gegebenenfalls als strategisch geführten Prozess aufzubauen. Zunächst einmal ist strategische Prozessführung neben politischen Interventionen ein zusätzliches Instrument, um gezielt gegen rechtliche Lücken im Menschenrechtsschutz vorzugehen und den

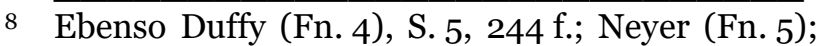
Open Society Justice Initiative (Fn. 5); siehe auch die entsprechende Relativierung in Südafrika bei Clark (Fn. 5). 
Betroffenen den Zugang zum Recht zu ermöglichen. Auch hat sich die Menschenrechtspraxis zu einem hochspezialisierten Rechtsgebiet ausdifferenziert, das von den Akteuren einen hohen Grad an Professionalisierung verlangt. Des Weiteren übersteigt die Komplexität der Fälle ebenso wie die damit verbundenen Kosten oftmals die Kapazität von Einzelpersonen und erfordert ein koordiniertes und institutionell abgestütztes Vorgehen.

\section{Behebung rechtlicher Lücken im Menschenrechtsschutz}

Die Schweiz weist generell einen hohen Schutzstandard auf. Dennoch darf dieser niemals als selbstverständlich gelten. Politische und gesellschaftliche Herausforderungen setzen die Menschenrechte immer wieder unter Druck. Auch weist die Umsetzung der Menschenrechte in der Schweiz einzelne Lücken auf. Sie betreffen sowohl die Umsetzung der Menschenrechte in einzelnen Rechtsgebieten als auch die praktischen Möglichkeiten ihrer effektiven Durchsetzung für den Einzelnen.

Inhaltlich bestehen in der Schweiz namentlich im Bereich der Rassendiskriminierung, des Asyl- und Ausländerrechts, der Gleichstellung der Geschlechter, der Rechte von Homosexuellen und gleichgeschlechtlichen Paaren, der häuslichen Gewalt, des Einsatzes von Gewalt durch die Polizei, der Rechte von Gefangenen, des Schutzes vor Überwachung und der Privatsphäre im Internet besondere Herausforderungen für den Menschenrechtsschutz. ${ }^{9}$ Verfahrensrechtlich bilden

9 Eine hilfreiche Übersicht geben die Studien des Schweizerischen Kompetenzzentrums für Menschenrechte und die Dokumente aus der Universal beispielsweise die qualifizierte Rügepflicht für Grundrechte (Art. 106 Abs. 2 BGG) ${ }^{10}$, die eingeschränkte Verfassungsgerichtsbarkeit für Bundesgesetze (Art. 190 BV) ${ }^{11}$ und die hohen Kosten (dazu hinten IV.4) bedeutende Hürden für den Menschenrechtsschutz.

15 Des Weiteren ist die Heterogenität der Rechtsprechung zum Menschenrechtsschutz auffällig. Einerseits haben Gerichte in vielen Fällen wegweisende Urteilsbegründungen zur Stärkung des Menschenrechtsschutzes vorgelegt. Das Bundesgericht und die Kantonsgerichte waren massgebend an der Entwicklung des Grundrechtsschutzes beteiligt. Exemplarisch dafür steht das Urteil des Bundesgerichts zum Recht auf Nothilfe ${ }^{12}$ oder zum Frauenstimmrecht. ${ }^{13}$ Andererseits ist auffällig, dass Menschenrechte insbesondere in zivil- und strafrechtlichen Verfahren oftmals nicht gerügt werden. Auch bleibt die grundrechtskonforme Auslegung der Rechtsgrundlagen durch die Gerichte teilweise aus oder erfolgt mit unzureichender Tiefe. ${ }^{14}$ Das macht es für die Betroffenen bis zu einem gewissen

Periodic Review der Schweiz vor dem Menschenrechtsrat der Vereinten Nationen.

1o $\mathrm{Zu}$ einer Kritik der Rügepflicht siehe etwa Jörg Paul Müller, Verwirklichung der Grundrechte nach Art. 35 BV, S. 130-136; siehe auch das Urteil des Bundesgerichts zum Verhüllungsverbot, in dem es sich mangels Rüge nicht mit der Verletzung der Religionsfreiheit befasst hat, BGE 144 I 281 E. 1.7.

11 Zur eingeschränkten Verfassungsgerichtsbarkeit einführend Ulrich Häfelin/Walter Haller/Helen Keller/Daniela Thurnherr, Schweizerisches Bundesstaatsrecht, 2016, N 273; Bernhard Waldmann, in: Basler Kommentar BV, Art. 35 N 31.

12 BGE 121 I 367.

13 BGE 116 Ia 359.

14 Siehe etwa BGE 140 I 305; kommentierend dazu Irene Grohsmann, Vaterschaftsurlaub, SKMRNewsletter Nr. 18 vom 13. November 2014. 
Grad unberechenbar, Grundrechte vor Gericht einzufordern. 15

Strategische Prozessführung bietet der Zivilgesellschaft ergänzend zu politischen Instrumenten ein hilfreiches rechtliches Instrument, um gezielt und koordiniert gegen rechtliche Lücken im Menschenrechtsschutz vorzugehen.

\section{Stärkung des Zugangs zum Recht bei Menschenrechtsverletzungen}

Neben materiellen Lücken im Grundrechtsschutz erschweren Lücken in der Rechtspraxis die Durchsetzung der Menschenrechte. Dazu gehört einerseits fehlendes Wissen der Betroffenen, wie sie ihre Rechte geltend machen können sowie fehlender Zugang zu niederschwelliger Rechtsberatung und zu professioneller Rechtsvertretung durch spezialisierte Anwältinnen und Anwälte. Andererseits dürfte in vielen Fällen die finanzielle Tragbarkeit von Rechtsberatung und professioneller Rechtsvertretung ein Hindernis für den Zugang zum Recht und damit auch für die Durchsetzung des Menschenrechtsschutzes im Einzelfall sein. ${ }^{16}$ Strategische Prozessführung ist

15 Siehe exemplarisch den vom Eidgenössischen Büro für Gleichstellung publizierten Bericht von Karine Lempen/Anne Voloder, Analyse der kantonalen Rechtsprechung nach dem Bundesgesetz über die Gleichstellung von Frau und Mann (2004-2015), 2017, der aufzeigt, wie unberechenbar und teuer es nach wie vor ist, die Geschlechtergleichstellung vor Gericht einzufordern; eine Studie des SKMR zeigt auf, dass generell deutlich weniger Frauen als Männer an Verfahren vor Bundesgericht beteiligt sind und grosser Forschungsbedarf im Bereich des gleichberechtigten Zugangs zur Justiz in der Schweiz besteht, Judith Wyttenbach/Eliane Braun, Weniger Frauen als Männer vor Bundesgericht, März 2019.

16 Die finanzielle Unterstützung in Grundrechtsfällen ist auf die unentgeltliche Rechtspflege in Gerichtsverfahren beschränkt. Rechtsberatung ohne Gerichtsverfahren ist nicht abgedeckt, ein Instrument, mit dem Einzelfälle gezielt unterstützt und damit den Betroffenen die Einforderung ihrer Rechte vor den Behörden ermöglicht werden kann.

\section{Spezialisierung und Professionali- sierung der Menschenrechtspraxis}

18 Der Menschenrechtsschutz hat sich in den vergangenen zwei Jahrzehnten zu einem spezialisierten Rechtsgebiet ausdifferenziert. Human Rights Litigation ist weltweit zu einem hochprofessionalisierten und umfangreichen Tätigkeitsgebiet geworden, an dem eine Vielzahl von Akteuren beteiligt sind. Das Volumen der Fälle und ihre normative und gesellschaftliche Tragweite haben zugenommen. Der Ausbau des Vertragssystems und die Anerkennung der Menschenrechte in bis anhin weniger thematisierten Gebieten hat für Menschenrechtsanliegen neue Foren geöffnet. ${ }^{17}$ Diese Entwicklung bedingt - vergleichbar mit den Veränderungen in anderen Rechtsbereichen eine personelle Spezialisierung und den Aufbau von Institutionen, die diese Komplexität $\mathrm{zu}$ bewältigen vermögen. Die zunehmende Häufigkeit professionell geführter strategischer Prozesse ist vermutungsweise Ausdruck dieser Entwicklung.
BGE 128 I 225, 231; Bernhard Waldmann, Basler Kommentar BV, Art. 29 BV N 66; Rhinow/ Schefer/Uebersax (Fn. 1), N 3051-3053b; kritische Würdigung des Rechtsschutzes bei Alexander Suter, Armut und Diskriminierung, 2015, N 138-141; Regina Kiener, Effektiver Zugang zur Justiz. Siehe etwa auch die Empfehlungen zur Stärkung niederschwelliger Rechtsberatung der Christoph Merian Stiftung, Soziale Handlungsfelder in der Stadt Basel, 2017, S. 45-48.

17 Übersichtlich Duffy (Fn. 4), S. 9. 


\section{Vernetzung der Akteure}

19 Historische Erfahrungen wie diejenige der Opfer fürsorgerischer Zwangsmassnahmen zeigen, wie stark die Durchsetzung der Menschenrechte unter politischem und gesellschaftlichem Druck erfolgt und wie isoliert die Betroffenen unter Umständen sein können. ${ }^{18}$ Menschenrechte schützen den Einzelnen gerade auch gegenüber demokratischen Mehrheitsentscheiden. Menschenrechtliche Interventionen erfolgen auch zum Schutz von Menschen mit gesellschaftlich unbeliebten Meinungen, Glaubensund Lebensformen. Menschenrechtsfälle erfolgen entsprechend oft vor dem Hintergrund einer politisch aufgeladenen Stimmung. Von einer einzelnen Person zu fordern, diesem Druck Stand zu halten, ist viel verlangt. Strategische Prozessführung erlaubt es, mittels Vernetzung die Isolation Einzelner zu mindern, den Einzelfall in einem gesellschaftlichen Gesamtkontext zu verorten, strukturelle Probleme aufzuzeigen und dadurch ein über den Einzelfall hinausweisendes Momentum zu erzeugen.

\section{Organisation und Finanzierung}

2o Strategische Prozessführung bedarf erfahrungsgemäss eines hohen Organisationsgrades, eines starken Netzwerks, umfangreicher finanzieller Ressourcen und einer umfassenden Kommunikationsstrategie. Diese Komponenten übersteigen in der Regel die Leistungsfähigkeit einer Einzelperson. Die Stärkung der Unterstützung von Betroffenen ebenso wie die Vernetzung von Anwältinnen und Anwälten, Universitäten und Nichtregierungsorganisationen entlastet die Be-

18 Siehe dazu ausführlich Vanessa Rüegger, Nichts als Sprache, myops 28/2016, S. 4-21. troffenen und macht den Fall handhabbarer. In den meisten Fällen, in denen Gerichte wegweisende Urteile zum Schutz der Menschenrechte aussprechen, gehen dem Urteil langjährige Öffentlichkeitsarbeit, intensive organisatorische Arbeit und umfangreiche Forschungstätigkeiten voraus. ${ }^{19}$ Ein koordiniertes Vorgehen im Rahmen strategischer Prozessführung kann einen wichtigen unterstützenden Beitrag leisten.

\section{Wie werden strategische Prozesse organisiert?}

21 Die Organisation strategischer Prozesse ist je nach Fall unterschiedlich. Akteure, Zielsetzung, gerichtliches Forum, Strategie und Finanzierung der Prozesse unterscheiden sich zuweilen bedeutend. In jedem Fall zentral ist die Auswahl eines geeigneten Falles, wobei auch ethische Fragen zu berücksichtigen sind. Die dynamische Entwicklung von Rechtsverfahren verunmöglicht es, strategische Prozesse zuverlässig planen zu können. Die Strategie sollte entsprechend regelmässig kritisch überprüft und angepasst werden. Auch ist eine begleitende Kommunikationsstrategie von grosser Bedeutung. Die Überprüfung, ob Prozesse die mit ihnen verfolgten Ziele auch tatsächlich erreichen, ist methodisch bedingt nur beschränkt möglich.

19 Beweis dafür ist beispielsweise die umfangreiche wissenschaftliche Literatur und rechtlichen Gutachten, die das Gericht zur Begründung seines für die Menschenrechte wegweisenden Urteile zitiert in BGE 121 I 367 (Anerkennung des Rechts auf Nothilfe) und BGE 116 Ia 359 (Anerkennung des Frauenstimmrechts). 


\section{Akteure}

22 In der Schweiz werden die meisten strategischen Prozesse ad hoc geführt. Im Ausland gibt es demgegenüber Institutionen, die auf die Führung von strategischen Menschenrechtsprozessen spezialisiert sind und langjährige Erfahrung vorweisen.

\section{a) In der Schweiz}

In der Schweiz gibt es momentan keine Organisation, die auf die strategische Prozessführung für Menschenrechtsfälle spezialisiert ist. Einzelne Organisationen oder Privatpersonen haben in der Vergangenheit ad hoc strategische Prozesse geführt. Viele der Fälle gehen auf die Initiative einer Einzelperson oder einer kleinen Gruppe an Personen zurück, die den Gerichtsfall gemeinsam mit einer Anwältin oder einem Anwalt mit hoher Bereitschaft zu persönlichem und oftmals unentgeltlichem Engagement, allenfalls unterstützt durch eine Rechtsschutzversicherung sowie Freunde und Familie, durchziehen. Bekannt sind die strategischen Prozesse von Ludwig Minelli, mit denen er sich für die Einhaltung der Menschenrechte in Strafverfahren und in der Haft einsetzte ${ }^{20}$ und damit auch bis an den EGMR gelangte. ${ }^{21}$ Weitere Beispiele sind weiter unten (V.) thematisiert.

24 Bis anhin verfolgen nur ganz wenige NGOs und Verbände eine systematische Strategie für die Führung strategischer Prozesse. Unter den Organisationen, die

20 BGE 99 Ia 262; BGE 100 Ia 378; BGE 102 Ia 201; BGE 102 Ia 279.

21 Urteil des EGMR Nr. 8660/79 vom 25. März 1983 (Minelli vs. Schweiz). Die Hintergründe des Falls sind beschrieben bei Adrian Riklin, Es begann mit einem simplen Branchentelefonbuch, in: Frau Huber geht nach Strassburg, (Fn. 1), S. 25-41. wiederholt strategische Prozesse geführt haben, finden sich namentlich die Demokratischen Juristinnen und Juristen Schweiz. Sie haben beispielsweise das Urteil zum Polizeigesetz Kanton Zürich ${ }^{22}$ erwirkt, ebenso wie das Urteil über die Kostentragungspflicht an Demonstrationen im Kanton Luzern ${ }^{23}$.

\section{b) In den USA}

25 Im Gegensatz zur Schweiz gibt es in den USA verschiedene Organisationen, die auf die strategische Prozessführung im Bereich des Menschenrechtsschutzes spezialisiert sind. Am bekanntesten sind die American Civil Liberties Union (ACLU), die National Association for the Advancement of Colored People (NAACP) und das Centre for Constitutional Rights (CCR). An dieser Stelle soll einzig die ACLU kurz vorgestellt werden.

26 Die ACLU entstand 1919 zur Unterstützung der Arbeitnehmerrechte. Sie entwickelte sich in den 1930er-Jahren zu einer Organisation, die sich für die in der Verfassung der Vereinigten Staaten gewährleisteten Bürgerrechte (Civil Rights) einsetzt. Seit 9/11 engagiert sich die ACLU auch für die Achtung der internationalen Menschenrechtsstandards innerhalb des amerikanischen Rechtssystems. Die ACLU ist dezentralisiert organisiert mit eigenen, unabhängigen Niederlassungen in 50 Staaten und hat 1.5 Millionen Mitglieder. Etwa 100 ACLU-Anwältinnen und Anwälte arbeiten landesweit mit rund 2000 Freiwilligen zusammen, um jährlich rund 2000 Fälle zu betreuen. Die Finanzierung erfolgt mittels Mitgliederbeiträgen und Spenden privater Stiftun-

22 BGE 136 I 87.

23 BGE 143 I 147. 
gen und Einzelpersonen. Unter den bekanntesten Prozessen der ACLU sind der Scopes-Prozess von $1925^{24}$ zur Stärkung der akademischen Freiheit, der 1954 gemeinsam mit der NAACP geführte Prozess Brown v. Board of Education ${ }^{25}$ gegen die Rassentrennung in öffentlichen Schulen und die Urteile in den 1970er-Jahren des Supreme Court in Roe v. Wade ${ }^{26}$ und Doe v. Bolton ${ }^{27}$ zur Legalisierung der Abtreibung. Jüngst hat die ACLU die Immigrationspolitik und die Notstandserklärung des amerikanischen Präsidenten Donald Trump angefochten. ${ }^{28}$

\section{c) In Deutschland}

27 Auch in Deutschland gibt es verschiedene Organisationen, die strategische Prozesse führen. Namentlich besteht seit 2007 mit dem European Center for Constitutional and Human Rights (ECCHR) eine Organisation, die sich auf die Durchsetzung der Menschenrechte mit juristischen Mitteln spezialisiert hat. Das ECCHR will konkrete Rechtsbrüche durch Regierungen, staatliche Akteure und Unternehmen juristisch aufarbeiten, die Rechtsansprüche der Betroffenen durchsetzen, strukturelles Unrecht offenlegen und durch begleitende Öffentlichkeitsarbeit die Zivilgesellschaft für Menschenrechtsverletzungen sensibilisieren. ${ }^{29}$ Das ECCHR

24 State of Tennessee v. John Thomas Scopes, Criminal Court of Tennessee (1925); Scopes v. State, 154 Tenn. 105 (1927).

25 Brown v. Board of Education, 347 U.S. 483 (1954).

26 Roe v. Wade, 410 U.S. 113 (1973).

27 Doe v. Bolton, 410 U.S. 179 (1973).

28 Die Geschichte der ACLU ist dargestellt in Judy Kutulas, The American Civil Liberties Union and the Making of Modern Liberalism, 2006; Robert C. Cottrell, Roger Nash Baldwin and the American Civil Liberties Union, 2000; Laura Weinrib, The Taming of Free Speech, 2016; Cole (Fn. 5).

29 Ich danke den Mitarbeitenden des ECCHR für die Auskunft über ihre Arbeit. führt regelmässig strategische Prozesse, gegenwärtig etwa vor den französischen Gerichten gegen das Unternehmen LafargeHolcim wegen Beihilfe zu Verbrechen gegen die Menschlichkeit in Syrien ${ }^{0}$, oder äussert sich im Rahmen von Amics Curiae Schriften zu laufenden Verfahren, beispielsweise beim International International Centre for the Settlement of Investment Disputes (ICSID) zur Verletzung von Menscherechten durch den Abbau von Gold in Rumänien. ${ }^{31}$

\section{d) Internationale Organisationen}

28 Eine der erfahrensten NGOs im Bereich der strategischen Prozessführung ist die Open Society Justice Initiative (OSJI), ein Programm der Open Society Foundation (OSF) mit Sitz in den USA und einem internationalen Aktionsradius. Die OSJI dokumentiert Verstösse, schlägt Lösungen vor, engagiert politische Entscheidungsträger und bietet seit 2003 rechtliche Unterstützung in strategisch geführten Prozessen. ${ }^{32}$ Die OSJI hat auch zahlreiche Fälle an den EGMR gebracht (siehe für ein Beispiel unten V.2). Neben der OSJI hat beispielsweise auch Amnesty International eine Abteilung zur Führung von strategischen Prozessen. 33

\section{Auswahl der Fälle}

29 Finanzielle und personelle Ressourcen sind nur beschränkt verfügbar. Strategische Prozessführung ist deshalb immer

$30 \overline{\text { European Center for Constitutional and Human }}$ Rights (ECCHR), Französisch-Schweizer Konzern: Verdacht der Beihilfe zu schwersten Verbrechen in Syrien.

31 (ECCHR), Umweltschutz versus Investitionsrecht - Der Fall der Goldmine in Rosia Montana Rumänien.

32 Open Society Foundation about the Open Society Justice Initiative.

33 Siehe Amnesty International, Strategic Litigation. 
mit der Frage nach der Auswahl der unterstützten Fälle verbunden. Die Wahl für oder gegen die Unterstützung eines Falles wirft schwierige ethische Fragen auf. Gerade dort, wo Spendengelder oder öffentliche Mittel zum Einsatz kommen, bedarf es einer Begründung, weshalb gerade dieser Einzelfall und nicht ein anderer die Unterstützung einer Organisation erhält.

Kriterien für die Wahl eines Falles können ganz unterschiedlich sein. Die Fallauswahl sollte sowohl die Bedeutung des Anliegens für die Betroffenen, die gesellschaftliche Tragweite der Rechtsfrage und die mögliche Wirkung eines Gerichtsverfahrens für den Menschenrechtsschutz berücksichtigen. So kann sich die Vertretung eines Falles aufdrängen, weil eine besonders schwere Menschenrechtsverletzung vorliegt oder die Situation dringlich ist. Das trifft beispielsweise auf Fälle im Asylverfahren zu, in denen etwa kleine Kinder von ihren Eltern getrennt werden. 34 Eine solche Trennung führt schon nach kürzester Zeit zu schweren psychischen Beeinträchtigungen für die Betroffenen.

Die Auswahl eines Falles kann aber auch damit motiviert sein, dass er sich besonders gut eignet, um eine Lücke im materiellen Recht $\mathrm{zu}$ thematisieren. So entschied sich Ruth Bader Ginsberg beispielsweise für eine strategische Prozessführung in einem unscheinbar anmutenden Fall über Sozialleistungen an einen Mann, der seine Mutter unterstützt. Der Fall eignete sich aber besonders gut dazu,

34

So beispielsweise im Urteil des Bundesgerichts 2C_1052/2016 vom 26. April 2017, dessen Verfahren mit Unterstützung von Amnesty Schweiz geführt wurde. auf die Ungleichstellung der Geschlechter in grundsätzlicher Weise aufmerksam zu machen, weshalb Bader Ginsberg den Fall gemeinsam mit der ACLU als strategischen Prozess führte. 35

32 Die Auswahl kann sich auch daraus ergeben, dass ein Fall stellvertretend für eine Vielzahl gleichartiger Fälle steht und deshalb mit einem Urteil eine breite Wirkung für viele Personen in ähnlichen Situationen erzielt werden kann. So hatte beispielsweise das Urteil Tarakhel ${ }^{6}$ zur Dublin-Rückführung einer Familie nach Italien nicht nur eine grosse Dringlichkeit für die Betroffenen, sondern auch Auswirkungen auf alle ähnlichen Fälle im europäischen Raum.

33 Ein weiteres Kriterium kann die Frage sein, wie geeignet eine Institution ist, um einen strategischen Fall zu führen, oder ob allenfalls eine andere Organisation besser geeignet ist, den Fall zu vertreten. Ebenfalls sollte geprüft werden, ob ein Gerichtsverfahren tatsächlich das richtige Mittel ist, um eine Lücke im Menschenrechtsschutz zu schliessen, oder ob sich nicht vielmehr alternative Konfliktlösungsmechanismen oder politische Arbeit besser eignen.

34 Strategische Prozessführung nutzt einzelne Gerichtsfälle, um auf die über den Einzelfall hinausweisende Tragweite der Rechtsfrage aufmerksam zu machen und

35 Die Geschichte von Ruth Bader Ginsberg und ihren strategischen Prozessen im Bereich der Geschlechtergleichstellung ist erzählt im Dokumentarfilm RBG (Betsy West/Julie Cohen, USA 2018) und im Spielfilm On the basis of sex (Mimi Leder, USA 2018). Siehe auch Ruth Bader Ginsberg, My own words, 2016; Cathleen Small, Ruth Bader Ginsburg, 2018.

36 Urteil des EGMR Nr. 29217/12 vom 4. November 2014 (Tarakhel vs. Schweiz). 
eine Verbesserung des Menschenrechtsschutzes für die gesamte Gesellschaft zu erzielen. Eine Ethik der Prozessführung verbietet es dabei, die Opfer von Menschenrechtsverletzungen zu instrumentalisieren und die Interessen der Allgemeinheit über diejenigen der Betroffenen zu stellen. Die Interessen der einzelnen Beschwerdeführerinnen und Beschwerdeführer, anhand deren Fall eine breitere Strategie aufgebaut wird, müssen stets im Zentrum des Verfahrens stehen. Anwältinnen und Anwälte von Betroffenen haben zudem eine ethische Verpflichtung, die Interessen ihrer Klientschaft konsequent und ohne Ausnahme über die gesellschaftlichen Interessen an einem Menschenrechtsfall zu stellen. Entscheidet eine Beschwerdeführerin oder ein Beschwerdeführer, dass er oder sie den Fall nicht weiterverfolgen oder ihren Fall nicht mehr mit einer grösseren Strategie verbinden möchte, so ist dieser Entscheid $\mathrm{zu}$ respektieren. Die Interessen der Beschwerdeführerinnen und Beschwerdeführer, ihre Entscheidungen und ihre Sicherheit und Integrität haben Priorität vor den strategischen Interessen, die mit ihrem Fall in Verbindung gebracht werden.

\section{Organisation der Prozesse}

35 Strategisch geführte Prozesse entwickeln sich dynamisch. Entsprechend anspruchsvoll ist ihre Planung. Weder ist ein «blueprint» für strategische Prozesse verfügbar, noch lässt sich die strategische Prozessführung für alle Phasen eines Prozesses lückenlos durchplanen. Vieles entscheidet sich ad hoc. Dennoch sollten strategische Prozess fortwährend in Bezug auf die Ziele, das Timing, die vorhandenen finanziellen und personellen Ressourcen, die Öffentlichkeitsarbeit und
Kommunikation, die Vernetzung mit anderen Organisationen und mögliche Hindernisse und Kritik überprüft werden.

36 Als Orientierung können folgende Punkte dienen:

- Identifikation von Zielen und Analyse möglicher Risiken;

- Finanzielle und persönliche Ressourcen;

- Vorbereitung des Falles und Wahl des Forums;

- Arbeit am Sachverhalt, der Beweisführung und der juristischen Argumentation;

- Einrahmung des Falles in eine Kommunikationsstrategie;

- Ergänzende Massnahmen ausserhalb des Gerichtsverfahrens (politische Arbeit und Lobbying, Öffentlichkeitsarbeit, Bildung, Monitoring);

- Mögliche Partnerinnen und Partner;

- Mögliche Gesprächspartnerinnen und -partner in Politik und Regierung;

- Alternative Konfliktlösungsmechanismen;

- Ergänzende Verfahren vor nationalen oder internationalen Gremien (zum Beispiel URP vor dem Menschenrechtsrat);

- Langfristige Ziele und mögliche Entwicklungen.

37 Als besonders wichtig in der Planung erweisen sich die Kommunikationsstrategie und der Umgang mit Erwartungen gegenüber den Parteien und in der 
Öffentlichkeit. Sämtliche beteiligten Akteure sollten ein realistisches Bild davon haben, welche Chancen und Risiken ein Gerichtsverfahren mit sich bringt und was mögliche persönliche und gesellschaftliche Auswirkungen des Falles sind. Was sich ausserhalb des Gerichts abspielt, ist für strategische Prozesse mindestens so wichtig wie die Entwicklung des Verfahrens selbst. Strategisch geführte Prozesse sind idealerweise mit einer professionell geführten Kommunikationsstrategie verbunden und beziehen die betroffenen Personen, Gruppen oder staatlichen Akteure aktiv mit ein. 37

\section{Finanzierung der Prozesse}

38 Die Finanzierung von Menschenrechtsfällen ist, unabhängig davon, ob sie als gewöhnlicher Einzelfall oder als strategischer Prozess geführt werden, mithin die grösste Herausforderung für die gerichtliche Durchsetzung der Menschenrechte..$^{8}$ Grundsätzlich obliegt es dem Staat sicherzustellen, dass der Zugang zur Justiz tatsächlich gewährleistet ist und nicht an der Finanzierung scheitert. Die Rechtsweggarantien verlangen nicht, dass der Rechtsweg kostenlos beschritten werden kann. Die Pflicht zur Leistung von Partei- und Prozesskosten ist aber eine rechtfertigungsbedürftige Einschränkung des Rechts auf Justizzugang. Einer Partei darf der Gerichtszugang nicht mit Verweis auf ökonomische Faktoren verwehrt werden. 39 Ganz zentral ist dabei auch eine funktionierende unentgeltliche Rechtspflege, darin eingeschlossen die unentgeltliche Rechtsverbeiständung.

Zum Ganzen ausführlich Duffy (Fn. 4).

Siehe auch Open Society Justice Initiative (Fn. 5).

39 Kiener (Fn. 2), 54 f.; Urteil des EGMR Nr. 8225/78 vom 28. Mai 1985 (Ashingdane v. UK), N 57.
39 Die Finanzierungsstrukturen für Menschenrechtsfälle sind in jedem Land unterschiedlich. In der Schweiz setzen sich die momentan zur Verfügung stehenden Finanzierungsquellen zusammen aus Eigenfinanzierung, Finanzierung durch Rechtsschutzversicherungen, staatliche Leistungen, Pro-Bono-Arbeit durch Anwältinnen und Anwälte und ein beschränktes Angebot an Unterstützung durch NGOs oder Trägervereinen. Neuere Formen der Finanzierung wie etwa Crowdfunding 40 sind in der Schweiz (noch) nicht verbreitet.

40 Während die Pluralität der Finanzierungsquellen grundsätzlich zu begrüssen ist, ist es in einer demokratischen Verfassungsordnung problematisch, wenn Menschenrechtsfälle an der Finanzierung scheitern. Obwohl keine statistischen Untersuchungen vorliegen, ist zu vermuten, dass Menschenrechtsfälle in besonders sensiblen Rechtsbereichen wie dem Asylwesen oftmals nur mit der Unterstützung von Pro-Bono-Leistungen der Anwältinnen und Anwälte, unentgeltlichen Rechtsberatungsangeboten und allenfalls finanzieller Unterstützung durch NGOs oder private Gönnerinnen und Gönner ans Gericht gelangen. Anders verhält es sich im Bereich der Wirtschaftsfreiheit oder der Eigentumsgarantie, wo Beschwerdeführer, beispielsweise Unternehmen, eher über die notwendigen Mittel verfügen dürften. Im Bereich des Strafrechts ist staatliche Unterstützung einfacher zugänglich und in vielen Fällen besteht ein Rechtsanspruch auf eine professionelle Rechtsvertretung. Demgegenüber fehlt diese Unterstützung

$4 0 \longdiv { \text { Innovativ in diesem Bereich beispielsweise die } }$ Plattform Crowdjustice. 
im Strafvollzug, was die Betroffenen empfindlich trifft.41

mögliche Finanzierungsquelle für Menschenrechtsfälle sind Pro-BonoDienste leistungsstarker Grosskanzleien. In den USA haben viele der grossen Wirtschaftskanzleien Pro-Bono-Abteilungen, mit denen sie einen Teil ihrer Dienstleistungen ohne Entgelt zur Verfügung stellen. Pro-Bono-Abteilungen grösserer Wirtschaftskanzleien im angelsächsischen Raum arbeiten in der Regel nicht direkt mit den Betroffenen, sondern mit NGOs oder Public Interest Law Firms zusammen. $4^{2}$ Die Geschichte von ProBono-Dienstleistungen führt weit zurück und ist eng mit dem philanthropischen Ethos des Anwalts als selbstloser Vertreter für die Forderung nach Recht und Gerechtigkeit verbunden. Pro-BonoDienstleistungen grosser Anwaltskanzleien stossen aber auch auf Kritik. Ihnen wird angelastet, dass sie lediglich der Reputations- und Imagepflege dienen, einer Elite erlauben, sich als selbstlos darzustellen, als Marketing-Tool für die Akquise von Klienten dienen, zur Entstaatlichung von Dienstleistungen beitragen, zu deren finanziellen Unterstützung der Staat verpflichtet wäre, oder defizitäre staatliche Finanzierungsstrukturen aufrechterhalten. 43

41 Siehe etwa Stephan Bernard, In der Schweiz haben nicht alle den gleichen Zugang zum Recht, NZZ am Sonntag vom 1. Februar 2020.

42 Ich danke den verschiedenen Pro-BonoPartnerinnen in New Yorker Wirtschaftskanzleien, die mir Auskunft über ihre Arbeit gegeben haben. $\mathrm{Zu}$ Pro-Bono siehe auch Alberto Alemanno/Lamin Khadar, Reinventing Legal Education, 2018, S. 20. Pro-Bono-Dienstleistungen und Pro-Bono-Praktiken sind etwa auch in Handbüchern anschaulich beschrieben, siehe beispielsweise PILNet, Pro Bono Handbook, 2015.

43 Siehe die entsprechende Kritik bei Charles Bosvieux-Onyekwelu, Law Firms and Lawyers'
42 Die europäische Pro-Bono-Bewegung ist nach wie vor im Aufbau. Auch lässt sich die staatliche Finanzierungsstruktur in vielen europäischen Ländern kaum mit der in dieser Hinsicht desolaten Situation in den USA vergleichen. In den meisten kontinentaleuropäischen Anwaltskanzleien ist Pro Bono nach wie vor keine institutionalisierte Praxis.44 Es bleibt zu prüfen, ob ein systematischeres ProBono-Engagement grösserer Kanzleien auch in der Schweiz einen Beitrag zur Stärkung des Menschenrechtsschutzes leisten könnte.

\section{Wirkung und Evaluation}

43 Die Frage nach messbaren Wirkungen strategischer Prozesse führt zu schwierigen methodischen Fragen. Obwohl in den vergangenen Jahren wichtige Erfahrungen mit quantitativen Analysen im Bereich der Rechtsanwendung gesammelt wurden, ist deren Genauigkeit und Aussagekraft nach wie vor umstritten. Qualitative Analysen über die Wirkung von Gerichtsfällen dürften praktisch eher machbar sein, weisen indessen die für solche Analysen üblichen Schwachstellen bezüglich ihrer Genauigkeit und verzerrenden Faktoren auf. Bei der strategischen Prozessführung kommt für die Analyse erschwerend dazu, dass sich die Wirkung eines Falles dynamisch im Verlaufe des Verfahrens entwickelt und sich nicht nur auf die Parteien auswirkt, sondern potenziell auch auf andere Interessengruppen, ebenso wie auf das Verhalten staatlicher Organe und politischer Akteure. Menschenrechtsfälle entstehen

Pro Bono Practice (beim Autor erhältlich), S. 4 f.; mit einem stärker abwägenden Standpunkt Lamin Khadar, The Growth of Pro Bono in Europe (PILnet Report), 2016.

44 Lamin Khadar (Fn. 43). 
meistens in Zusammenarbeit oder in einem Netzwerk mit zivilgesellschaftlichen Organisationen und Interessengruppen. Oftmals bleibt unklar, auf welche Initiative beispielsweise eine politische Reaktion zurückzuführen ist. Strategische Prozessführung greift meist Themen auf, die ohnehin bereits seit längerem in einem breiten Kontext diskutiert werden. Welchen Beitrag ein Gerichtsverfahren ganz konkret zu grossen gesellschaftlichen Veränderungen leistet, ist schwierig bis unmöglich zu beurteilen. 45

44 Des Weiteren ist wichtig zu verstehen, dass die Wirkung eines Gerichtsfalls nicht mit dem Inhalt des Urteils gleichgesetzt werden darf. Ein verlorener Fall kann unter Umständen einen wichtigen Beitrag zur Unterstützung eines Anliegens leisten («success without victory»), beispielsweise indem ein politischer Prozess angeregt wird. Genauso kann ein gewonnenes Urteil keine oder negative Auswirkungen haben («victory without success»). Keine Auswirkungen hat ein gewonnenes Urteil beispielsweise, wenn es von den Behörden nicht umgesetzt wird. Negative Auswirkungen hat ein Urteil, wenn es zu vermehrter Gewalt und Diskriminierung, einem politischen Rückschlag oder einer Schwächung der Legitimation der Gerichte führt. 46 Auch wenn die Wirkung strategischer Prozessführung zur Durchsetzung der Menschenrechte also kaum messbar ist, sollten die einzelnen Verfahren dennoch von sorgfältiger Reflexion und Evaluation begleitet sein. 47

45 Duffy (Fn. 4), S. 37 ff.

46 Zum Ganzen im amerikanischen Kontext Lobel (Fn. 5).

47 Für weiterführende Hinweise siehe Duffy (Fn. 4), S. 46.

\section{Welche Beispiele für strategische Prozesse gibt es?}

45 Beispiele für strategische Prozessführung sind zahlreich. Prominente Beispiele finden sich sowohl bei den innerstaatlichen Gerichten verschiedener Länder als auch vor internationalen Gerichten. Nachfolgend sollen fünf Fälle exemplarisch dargestellt werden. Der Fall Grootboom vor dem südafrikanischen Verfassungsgericht gilt in der Literatur als einer der wegweisenden strategisch geführten Prozesse. Der Fall D.H. v. The Czech Republic ist ein von einer Koalition von NGOs in intensivem Dialog mit den Betroffenen geführter Prozess, der gut dokumentiert und evaluiert ist. Aus der Schweiz sind drei jüngere Prozesse dargestellt, die je unterschiedlich organisiert sind und zugleich auf je eigene Weise die Ausdifferenzierung strategischer Prozessführung im Kontext der Schweizer Rechtsordnung andeuten.

\section{Government of South Africa}

\section{v. Grootboom}

46 Der international vielleicht bekannteste strategisch geführte Fall ist Government of South Africa v. Grootboom ${ }^{48}$, welcher mit einem Urteil des südafrikanischen Verfassungsgerichts endete. Die Beschwerdeführerin war aus ihrer informellen Behausung vertrieben worden, die sich auf privatem Land befand. Dieses wurde geräumt, weil es für staatlich finanzierte Häuser vorgesehen war. Die Beschwerdeführerin beantragte, dass ihr die Regierung gestützt auf das in der südafrikanischen Verfassung gewährleistete Right to Housing eine angemessene

48 South African Constitutional Court 2001 (1) SA 46 (CC) from 4. October 2009 (Government of South Africa v. Grootboom). 
Unterkunft zur Verfügung stellt, bis sie in eine dauerhafte Unterkunft umziehen kann. Das Verfassungsgericht bestätigte im wegweisenden Fall die Justiziabilität wirtschaftlicher und sozialer Rechte und erläuterte die Besonderheiten der staatlichen Verpflichtungen für diese Rechte. Es befand ferner, dass der Staat verpflichtet ist, unmittelbare positive Massnahmen zu ergreifen, um die minimalen Bedürfnisse von Menschen in Armut und Not zu decken.

Der Grootboom-Fall gilt als Testfall für strategische Prozessführung, weil er mit fachlicher und finanzieller Unterstützung von NGOs geführt wurde und in eine grössere Kommunikationsstrategie zu Fragen der Justiziabilität der wirtschaftlichen und sozialen Rechte und den staatlichen Leistungspflichten eingebettet war. Das Urteil war insofern ein Erfolg, als das Gericht viele der Vorbringen im Sinne der Beschwerdeführerin beurteilte. Auf die Lebenssituation der Beschwerdeführerin hatte das Urteil indessen keinerlei Auswirkungen. Sie verstarb in Armut und ohne je die Unterstützung staatlich subventionierten Wohnraums erhalten zu haben. 49

\section{D.H. v. The Czech Republic}

48 Ein Beispiel aus dem europäischen Kontext ist das Urteil D.H. v. The Czech Republic50 des Europäischen Gerichtshofs für Menschenrechte. In verschiedenen Ländern Osteuropas wiesen Richtlinien und Praktiken Mitglieder der Roma seit

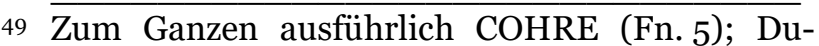
gard/Langford (Fn. 5); Sandra Liebenberg, Socioeconomic rights, Adjudication under a transformative constitution, 2010.

50 Urteil des EGMR Nr. 57325/oo vom 13. November 2007 (D.H. v. The Czech Republic).
Generationen getrennten öffentlichen Schulen zu. Ab Ende der 1990er-Jahren begannen die Roma sich mit Gerichtsverfahren gegen die getrennte Zuteilung zu wehren. Nach einer Reihe von Urteilen und Berufungen vor nationalen Gerichten verurteilte der Europäische Gerichtshof für Menschenrechte (EMRK) mit dem Urteil D.H. v. The Czech Republic die Praxis als Rassendiskriminierung.

49 Die Beschwerdeführer wurden massgebend von nationalen und internationalen NGOs unterstützt, so auch bei der aufwändigen Beweisführung, und hatten international anerkannte Menschenrechtsanwältinnen und -anwälte als professionelle Rechtsvertretung zur Seite. ${ }^{1}$ Die systematische Auswertung des Urteils legt offen, dass die strategische Prozessführung im Fall D.H. v. The Czech Republic und in ähnlichen Fällen nicht nur ein wegweisendes Urteil, sondern auch bedeutende Änderungen in Politik und Praxis zu bewirken vermag. Die Auswertung ist aber auch ernüchternd, was die effektive Macht der Gerichte gegenüber anderen staatlichen Gewalten und sozialen Praktiken angeht. Auch liess sich im beobachteten Zeitraum das Aufkommen neuer Formen der Diskriminierung beobachten. ${ }^{2}$ Die Studie zeigt in gewissem Sinne auf, was sich für strategische Prozessführung generell abzeichnet: Strategische Prozesse können einerseits wirkungsvoll sein, aber auch unbeabsichtigte Folgen haben.

\section{Vaterschaftsurlaub}

50 Auch in der Schweiz finden sich verschiedene Beispiele für strategisch ge-

51 Open Society Justice Initiative (Fn. 5), S. 21 f., 29.

52 Open Society Justice Initiative (Fn. 5), S. 33 ff. 
führte Prozesse (siehe auch IV.1.a). Ein jüngeres Beispiel ist das Urteil des Bundesgerichts zum Vaterschaftsurlaub.53 Der Beschwerdeführer wurde im Sommer 2012 zum zweiten Mal Vater. Mit Unterstützung seines Arbeitgebers stellte er bei der zuständigen AHV-Zweigstelle Antrag auf Elternschaftsentschädigung für 6 Wochen zum Prozentsatz, welcher weiblichen Angestellten gewährt wird. Die Ausgleichskasse des Kantons Bern verneinte einen Anspruch auf Entschädigung, ebenso wie das Verwaltungsgericht des Kantons Bern. Das Bundesgericht konnte darin keine Diskriminierung erkennen und wies die Beschwerde ab.

Der Fall ist ein interessantes Beispiel, weil er aufzeigt, wie ein Fall bewusst für die Mobilisierung für ein gesellschaftliches Anliegen verwendet werden kann und wie wichtig dabei eine breit abgestützte Trägerschaft und die Vernetzung der Akteure sind. Die Verfahrenskosten wurden zum grösseren Teil von einem von Freunden gegründeten Verein getragen. Die Anwältin leistete ihre Arbeit pro bono. Der Verein wurde von männer.ch und alliance $\mathrm{F}$, sowie von Vertreterinnen und Vertretern aller grossen Parteien, dem Kinderarzt Remo Largo und weiteren unterstützt. Das Verfahren war trotz negativem Entscheid dennoch insofern erfolgreich, als es breite Kritik und eine öffentliche Diskussion auslöste und damit auch das Rechtsetzungsverfahren dynamisierte. 54

\section{Behindertengerechte öffentliche Verkehrsmittel}

52 Der Dachverband Inclusion Handicap hatte im Januar 2018 Beschwerde gegen die befristete Betriebsbewilligung der Dosto-Züge der SBB eingereicht, weil diese von Menschen mit Behinderungen nicht selbständig genutzt werden könnten. Im November 2018 einigten sich Inclusion Handicap und die SBB aussergerichtlich zu vier Punkten. Kurz danach entschied das Bundesverwaltungsgericht, zehn der elf verbleibenden Rechtsbegehren abzuweisen.55 Der Fall ist vor Bundesgericht hängig.

53 Der Streit um die neuen SBB-Züge zog sich über Jahre hinweg und erreichte einen Umfang, der von einer einzelnen Person alleine kaum zu bewältigen gewesen wäre, gerade auch was das finanzielle Risiko betrifft. Die relativ kleine NGO sah sich einem der grössten öffentlichen Unternehmen gegenüber mit entsprechenden personellen und finanziellen Mitteln für ihre Rechtsvertretung. Die komplizierten Verhandlungen und Beweisführungsverfahren, die umfangreichen Beschwerdeschriften und die aufwändige Medien- und Kommunikationsarbeit wären ohne die Unterstützung des Verbandes, den Einbezug von externen Menschenrechtsexpertinnen und experten und einer starken institutionellen Vernetzung kaum zu bewerkstelligen gewesen. ${ }^{6}$
53 BGE 140 I 305.

54 Ich danke Charlotte Sieber-Gasser für die Auskunft zum Fall.
55 Urteil des Bundesverwaltungsgerichts A-359/2018 vom 20. November 2018.

56 Ich danke Caroline Hess-Klein, Martin Looser und Markus Schefer für die Auskunft zum Fall. 


\section{Racial Profiling}

54 Ein weiteres aktuelles Beispiel für eine strategische Prozessführung ist der Fall Wa Baile, mit dem die Praktik des Racial Profilings thematisiert wird. Wa Baile hatte sich im Februar 2015 im Rahmen einer polizeilichen Personenkontrolle am Zürcher Hauptbahnhof geweigert, seinen Ausweis vorzuweisen, weil er die Kontrolle als rassistisch empfand. Wa Baile entschied sich, den darauf erlassenen Strafbefehl anzufechten, um sich für eine Schweiz zu engagieren, in der alle unabhängig von ihrer Hautfarbe gleich behandelt werden. Am 7. März 2018 hat das Bundesgericht die Verurteilung von Mohamed Wa Baile durch das Zürcher Obergericht bestätigt.57 Der Fall ist momentan vor dem EGMR hängig.

Das Verfahren wird von einem breiten Kreis von Fachpersonen unterstützt und als strategischer Prozess gegen das Racial Profiling geführt. Das Strafverfahren soll über den Einzelfall hinaus Diskriminierungen in der Schweiz bekämpfen, dem Thema die nötige Aufmerksamkeit verleihen, die Bevölkerung sensibilisieren und Betroffene zu eigenem Handeln ermächtigen. 58

\section{Plädoyer für eine Stärkung der Menschenrechtspraxis}

Der Menschenrechtsschutz hat sich in den vergangenen Jahrzehnten zu einem hochspezialisierten Rechtsgebiet ausdifferenziert. Unter diesen veränderten Be-

57 Urteil des Bundesgerichts 6B_1174/2017 vom 7. März 2018.

58 Zum Fall siehe ausführlich die Informationsplattform humanrights, Rassistisches Profiling: Bundesgericht bestätigt Urteil im Fall «Wa Baile». Ich danke Stephan Bernard und Tarek Naguib für die Auskunft zum Fall. dingungen bedürfen die Rechtsberatung, die Rechtsvertretung und die Rechtsprechung im Bereich der Menschenrechte der Spezialisierung und Professionalisierung. Spezifisches Fachwissen zu den Menschenrechten besteht in der Schweiz bis anhin hauptsächlich an den Universitäten. Es ist nur punktuell und ad hoc mit der praktischen Ausbildung sowie der Rechtsberatung und Rechtsvertretung verknüpft.

57 In vielen Demokratien bestehen Organisationen und Ausbildungen, die eine fachliche Spezialisierung von Anwältinnen und Anwälten gewährleisten und Betroffene bei der Durchsetzung ihrer Menschenrechte vor Gericht beraten und unterstützen. In den USA, einer der ältesten Demokratien, besteht mit der American Civil Liberties Union (ACLU) beispielsweise eine Organisation, die sich mittels Ausbildung und Informationsaustausch für den präventiven Schutz der Grundrechte einsetzt und mit einer spezialisierten Rechtsvertretung Betroffene von Menschenrechtsverletzungen vor Gericht unterstützt. Sie arbeiten dabei mit Universitäten und Anwaltskanzleien zusammen. Die amerikanischen Universitäten bieten ihrerseits Kurse an, in denen die Studierenden eine praktische Ausbildung zur Durchsetzung der Menschenrechte erhalten (sogenannte human rights clinics). Entsprechende Organisationen und Ausbildungsformen gewinnen auch in Europa an Bedeutung.

58 In den vergangenen Jahren haben viele Schweizer Universitäten Menschenrechtskliniken gegründet. Auch die Organisation strategischer Prozessführung durch die Zivilgesellschaft professionalisiert sich. Das Entwicklungspotenzial ist 
in der Schweizer Rechtspraxis jedoch noch bei Weitem nicht ausgeschöpft. Juristinnen und Juristen bedürfen einer spezialisierten Ausbildung, um Menschenrechte als normativ anspruchsvolles Querschnittgebiet in der gesamten Rechtsordnung und unter herausfordernden politischen und rechtlichen Umständen zur Anwendung zu bringen. Ziel eines effektiven Grundrechtsschutzes muss es sein, einen Ausbildungsstandard und ein Angebot in der Rechtspraxis zu erreichen, das der fundamentalen Bedeutung der Menschenrechte, ihrer anspruchsvollen Rechtsdogmatik, ihrer herausforderungsreichen praktischen Anwendung und dem hohen Spezialisierungsgrad des Rechtsgebiets gerecht wird. Dazu gehört auch eine stärkere Institutionalisierung im Bereich der strategischen Prozessführung. Dabei gilt es stets zu bedenken, dass strategische Prozessführung kein Allheilmittel ist. Strategic Human Rights Litigation ist ein Instrument, das unter den richtigen Umständen und in Kombination mit anderen Mitteln zur Stärkung der Menschenrechte beitragen kann, dies aber nicht zwingend tut. 